\title{
B Lymphoblastic Leukemia/Lymphoma with Hypodiploidy
}

National Cancer Institute

\section{Source}

National Cancer Institute. B Lymphoblastic Leukemia/Lymphoma with Hypodiploidy. NCI Thesaurus. Code C80338.

A precursor lymphoid neoplasm composed of B-lymphoblasts which contain less than 46 chromosomes. It has an unfavorable clinical outcome. 